\title{
Undersampled Cine 3D tagging for rapid assessment of cardiac motion
}

\author{
Christian T Stoeck ${ }^{1}$, Robert Manka ${ }^{1,2}$, Peter Boesiger ${ }^{1}$ and Sebastian Kozerke ${ }^{1,3^{*}}$
}

\begin{abstract}
Background: CMR allows investigating cardiac contraction, rotation and torsion non-invasively by the use of tagging sequences. Three-dimensional tagging has been proposed to cover the whole-heart but data acquisition requires three consecutive breath holds and hence demands considerable patient cooperation. In this study we have implemented and studied $\mathrm{k}$-t undersampled cine 3D tagging in conjunction with $\mathrm{k}$-t PCA reconstruction to potentially permit for single breath-hold acquisitions.

Methods: The performance of undersampled cine 3D tagging was investigated using computer simulations and in-vivo measurements in 8 healthy subjects and 5 patients with myocardial infarction. Fully sampled data was obtained and compared to retrospectively and prospectively undersampled acquisitions. Fully sampled data was acquired in three consecutive breath holds. Prospectively undersampled data was obtained within a single breath hold. Based on harmonic phase (HARP) analysis, circumferential shortening, rotation and torsion were compared between fully sampled and undersampled data using Bland-Altman and linear regression analysis.

Results: In computer simulations, the error for circumferential shortening was $2.8 \pm 2.3 \%$ and $2.7 \pm 2.1 \%$ for undersampling rates of $\mathrm{R}=3$ and 4 respectively. Errors in ventricular rotation were $2.5 \pm 1.9 \%$ and $3.0 \pm 2.2 \%$ for $R=3$ and 4 . Comparison of results from fully sampled in-vivo data acquired with prospectively undersampled acquisitions showed a mean difference in circumferential shortening of $-0.14 \pm 5.18 \%$ and $0.71 \pm 6.16 \%$ for $R=3$ and 4 . The mean differences in rotation were $0.44 \pm 1.8^{\circ}$ and $0.73 \pm 1.67^{\circ}$ for $R=3$ and 4 , respectively. In patients peak, circumferential shortening was significantly reduced ( $p<0.002$ for all patients) in regions with late gadolinium enhancement.
\end{abstract}

Conclusion: Undersampled cine 3D tagging enables significant reduction in scan time of whole-heart tagging and facilitates quantification of shortening, rotation and torsion of the left ventricle without adding significant errors compared to previous 3D tagging approaches.

Keywords: Motion analysis, Myocardial tagging, k-t undersampling, Principal component analysis, Image reconstruction

\section{Background}

Patients with myocardial infarction (MI), coronary artery disease $(C A D)$ or impaired signal conduction in the heart such as left bundle branch block (LBBB) suffer from reduced cardiac function. In patients undergoing cardiac resynchronization therapy (CRT) [1] it is of great interest to generate a mechanical map indicating local

\footnotetext{
* Correspondence: kozerke@biomed.ee.ethz.ch

'Institute for Biomedical Engineering, University and ETH Zurich, Gloriastrasse 35, 8092 Zurich, Switzerland

${ }^{3}$ Imaging Sciences and Biomedical Engineering, King's College London, London, United Kingdom

Full list of author information is available at the end of the article
}

dyssynchrony [2]. Among the various mechanical parameters, cardiac strain and torsion have been shown valuable clinical parameters in patients with MI [3] or aortic stenosis [4,5]. Cardiovascular magnetic resonance (CMR) offers a functional imaging modality to assess cardiac motion pattern and synchrony of contraction $[6,7]$ non-invasively.

Up to date multiple approaches have been used to investigate myocardial motion such as displacement encoding with stimulated echo (DENSE) [8,9], velocity encoding [10-12], tagging by spatial modulation of magnetization (SPAMM) [13] and complementary 
spatial modulation of magnetization (CSPAMM) [14]. These methods can either be applied in conjunction with two dimensional (2D) or three dimensional (3D) imaging. In 2D acquisitions, multiple slices are imaged along the left ventricle (LV) and strain maps are calculated slice-by-slice. Two-dimensional acquisitions require additional techniques in order to compensate for through-plane motion. To this end, slice following $[15,16]$, acquisition of additional orthogonal slices [17] or the encoding of through-plane displacement $[18,19]$ (zHARP) have been proposed. While reconstruction of 3D strain patterns from two bi-planar acquisitions does require interpolation, slice-following techniques provide only a projection of true $3 \mathrm{D}$ motion onto a $2 \mathrm{D}$ subspace. Using zHARP additional gradients in through-slice direction are applied to estimate through-plane displacement by solving a set of linear equations [18].

To circumvent the need for interpolation and slicefollowing techniques, true 3D tagging has been proposed [20]. By applying modulation of magnetization in all three spatial directions, through-plane motion is intrinsically captured and hence no slice-following is needed. Furthermore, 3D acquisition provides full LV coverage and yields an intrinsically higher signal-to-noise (SNR) compared to 2D imaging. While cine 2D acquisitions easily fit into a single breath hold, 3D acquisitions require multiple breath holds with long duration [20]. Further approaches attempted to shorten scan time to four breath holds of $21 \mathrm{R}-\mathrm{R}$ intervals each [21] by only sampling around the harmonic peaks in k-space. These efforts, however, led to a lower spatial resolution in the two phase encoding directions. A different approach was proposed by Zhong et al. [22] using a 3D DENSE acquisition covering the entire left ventricle during free breathing. However, acquisition durations of up to 20 min [23] depending on navigator efficiency were reported and hence feasibility for routine clinical use may be questioned. Rutz et al. [24] introduced an accelerated 3D CSPAMM method only requiring three navigator gated breath holds of $18 \mathrm{R}-\mathrm{R}$ intervals each. This implementation was tested in a clinical study with patients suffering from MI and LBBB [25] providing 3D maps of synchrony and magnitude of contraction. To address issues of different breath hold levels the data obtained from respiratory navigators was used to correct for potential offsets. In their study the respiratory navigator was placed onto the right hemi-diaphragm and thereby respiratory induced displacement of the heart was approximated according to a linear relationship between liver and heart displacement [26]. The accuracy of the linear translation from motion of the lung liver interface to the position of the heart is still being debated. Nehrke et al. [27] reported a strong correlation of the displacement of the right hemi-diaphragm and the heart, but also found significant subject dependent variability in the correction coefficients especially comparing inspiration and expiration. Subject dependency of the translation of breathing induced liver motion to bulk motion of the heart has been confirmed by Moghari et al. [28].

In general, data acquisition can be accelerated by undersampling in spatial and temporal dimensions. Among the various approaches, two strategies have gained significant attention. Compressed sensing (CS) [29] employs non-linear reconstruction methods to recover information from randomly or pseudo-randomly undersampled data. Inherently, compressed sensing algorithms require incoherent sampling and hence become applicable if a sufficient number of phase-encodes exists. In case of one-dimensional tagging preparation, the number of Cartesian phase-encodes orthogonal to the tag direction can be greatly reduced providing an efficient and simple way for scan time reduction. In consequence the degrees of freedom to generate random sampling patterns become very limited and the application of compressed sensing appears less favourable in this particular application.

The second acceleration strategy involves uniform spatiotemporal undersampling in conjunction with linear reconstruction algorithms. In k-t BLAST and k-t SENSE [30] low spatial but full temporal resolution training data is used to unfold signal aliasing resulting from data undersampling. The drawback of these methods relates to temporal filtering if undersampling rates increase. To address this issue, Principal Component Analysis (PCA) of the spatial-temporal frequency domain data was introduced and results obtained with $\mathrm{k}$-t PCA show improved temporal fidelity [31].

Although $\mathrm{k}$-t undersampling has been extensively applied in CMR including cine and real-time imaging [32,33], perfusion [34-39] and phase contrast imaging [40-42], only few attempts of applying k-t undersampling to tagging have been reported [43,44].

The objective of the present study was to implement and test $\mathrm{k}-\mathrm{t}$ undersampled whole-heart 3D CSPAMM tagging for rapid assessment of cardiac motion. The performance is demonstrated on simulated data, data obtained in healthy subjects and in patients with myocardial infarction.

\section{Methods}

k-t PCA

To reconstruct undersampled 3D tagging data, k-t PCA [31] is used. Similar to k-t BLAST [30], acquired data is divided into 1) training data with low spatial resolution (in phase encoding directions $\mathrm{k}_{\mathrm{y}} / \mathrm{k}_{\mathrm{z}}$ ) but full temporal resolution and 2) k-t undersampled data with high spatial resolution and full temporal resolution. The 
training data $p_{\text {train }}(x, t)$ is Fourier transformed to be represented in the spatial-temporal frequency domain $(x-f)$. Using principal component analysis $p_{\text {train }}(x, f)$ is then decomposed into a basis of temporally dependent functions $b\left(f_{j}\right)$ corresponding to the principal components (pc) and spatially dependent weighting coefficients $w_{\text {train }}(x)$ in $\mathrm{x}$-pc space according to:

$$
p_{\text {train }}\left(x_{i}, f_{j}\right)=\vec{b}\left(f_{j}\right) \vec{w}_{\text {train }}\left(x_{i}\right)^{T}
$$

The aliased signal at point $\left(x, f_{j}\right)$ resulting from $R$-fold undersampling can be written as:

$$
p_{\text {alias }}\left(x, f_{j}\right)=\sum_{i=1}^{R} \vec{b}\left(f_{j}\right) \vec{w}\left(x_{i}\right)^{T}
$$

with $w\left(x_{i}\right)$ denoting the spatial weighting coefficients of the unaliased image. Hence the aliased signals $P_{\text {alias }}(x, f)$ can be expressed as:

$$
\begin{aligned}
P_{\text {alias }} & =\left[\begin{array}{c}
p_{\text {alias }}\left(x, f_{1}\right) \\
p_{\text {alias }}\left(x, f_{2}\right) \\
\ldots \\
p_{\text {alias }}\left(x, f_{N}\right)
\end{array}\right]=E \vec{w}_{x} \text { with } \vec{w}_{x} \\
& =\left[\begin{array}{c}
\vec{w}^{T}\left(x_{1}\right) \\
\vec{w}^{T}\left(x_{2}\right) \\
\ldots \\
\vec{w}^{T}\left(x_{R}\right)
\end{array}\right] \text { and } E=\left[\begin{array}{c}
\vec{b}\left(f_{1}\right) \\
\vec{b}\left(f_{2}\right) \\
\ldots \\
\vec{b}\left(f_{N}\right)
\end{array}\right]
\end{aligned}
$$

Finally, the unaliased spatial weighting coefficients are obtained by solving:

$$
w_{x}=\Theta E^{H}\left(E \Theta E^{H}+\psi\right)^{+} P_{\text {alias }}
$$

where $\Theta$ represents an estimate of $w_{x}$ from training data, $\Psi$ denotes noise variance, $H$ conjugate transpose and + the Moore-Penrose pseudo-inverse.

\section{Computer simulation}

All simulations were performed in Matlab (The MathWorks, Natick, MA, USA). Three orthogonal stacks with line tagging modulation in readout direction were generated (Figure 1a). The CSPAMM method [14] was simulated to avoid tag line fading. The model consisted of a contracting left ventricle as well as static tissue representing chest wall and liver. Circumferential shortening and rotation as measured in a healthy subject at basal and apical level was linearly interpolated along the longaxis to create three-dimensional motion data. Peak circumferential shortening was $18.8 \%$ and $17.8 \%$ for base and apex, respectively. Peak rotation was $-3.2^{\circ}$ and $10.3^{\circ}$ for base and apex (Figure 1b-d). Longitudinal shortening obtained from the same in-vivo subject was incorporated. Simulations of undersampled data acquisition were compared to fully sampled simulated data sets with equivalent spatial and temporal resolution. The matrix

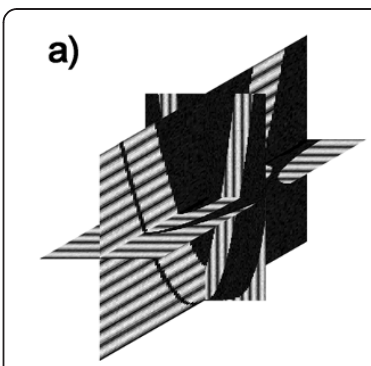

b)
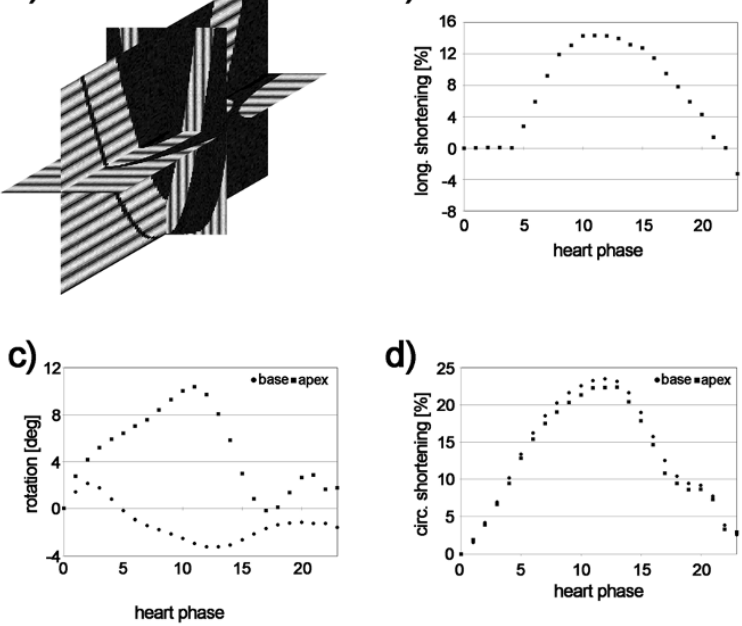

d)

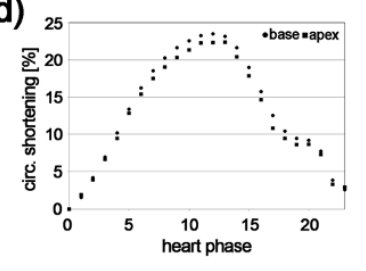

Figure 1 Numerical simulation. Three stacks with orthogonal line tag pattern were simulated (a). As input for the model, longitudinal shortening (b), rotation (c) and circumferential shortening (d) obtained from one in-vivo acquisition were used.

size was set according to practical values [24,25] (Table 1). Gaussian noise was added to k-space data before undersampling resulting in a SNR of 25 prior to undersampling. Both undersampled and training data were extracted from the computer model (Figure 2b). Undersampling rates of $\mathrm{R}=3,4,5$ and 8 were simulated. In all simulations, five training profiles were used in $\mathrm{k}_{\mathrm{y}}$ and $\mathrm{k}_{\mathrm{z}}$ direction resulting in a total of 25 training profiles.

In order to study regional wall motion abnormalities, myocardial infarctions of different severity were simulated. To this end, radial shortening in the lateral sector was changed from $100 \%$ (no infarction) to $0 \%$ (completely static) in steps of $10 \%$ (Figure 3 ). The reduction of myocardial motion in the infarcted zone was applied transmurally along the entire long axis of the left ventricle. Reduction of radial shortening directly reduced the circumferential contraction. In order to assure a smooth transition between infarcted and healthy tissue, the infarcted tissue was continuously "attached" to the adjacent healthy tissue, by reducing the motion damping factor continuously over a sector of $40^{\circ}$ on both ends of the infarcted region.

\section{In-vivo measurements}

Eight healthy subjects ( 7 male, age: $27.5 \pm 3.5$ years) and five patients with myocardial infarction (5 male, age: $54.8 \pm 5.9$ years, 1 patient with acute myocardial infarction post percutaneous coronary intervention (PCI), 4 patients with chronic myocardial infarction) were studied. Imaging was performed on a $1.5 \mathrm{~T}$ Philips Achieva System (Philips Healthcare, Best, the Netherlands) using 
Table 1 Parameters used for numerical simulation

\begin{tabular}{lccccc}
\hline Undersampling factor & Matrix size $\mathbf{k}_{\mathbf{x}} \times \mathbf{k}_{\mathbf{y}}$ & Slices $\mathbf{k}_{\mathbf{z}}$ & Heart phases & Training profiles $\left(\mathbf{k}_{\mathbf{y}} \times \mathbf{k}_{\mathbf{z}}\right)$ & $\mathbf{R}_{\mathbf{n e t}}$ \\
\hline 3 & $65 \times 15$ & 15 & 24 & $25(5 \times 5)$ & 2.3 \\
\hline 4 & $65 \times 20$ & 15 & 24 & $25(5 \times 5)$ & 3 \\
\hline 5 & $65 \times 20$ & 15 & 24 & $25(5 \times 5)$ & 3.5 \\
\hline 8 & $65 \times 16$ & 15 & 24 & $25(5 \times 5)$ & 4.4 \\
\hline
\end{tabular}

a 5 channel cardiac receiver array. Written informed consent was obtained from all subjects and the protocol was approved by the institutional review and ethics boards.

Tagged images were acquired using an ECG triggered multi-shot EPI sequence with sequence parameters listed in Table 2. As illustrated in the sequence diagram in Figure 2 the tagging preparation was applied within $10 \mathrm{~ms}$ after the detection of the R-wave. As the first RF pulse used for tagging preparation was applied selectively in phase encoding direction, signal outside the field-of-view was suppressed after CSPAMM subtraction and hence reduced field-of-view acquisition could be performed [16]. The imaging sequence was repeated to acquire three orthogonally tagged stacks. For each stack 21-24 heart phases were recorded.

Fully sampled data was acquired in three consecutive breath holds. To guarantee similar breath hold position for each tagged stack a pencil beam respiratory navigator placed on the right hemi-diaphragm was used. The position of the lung liver interface was monitored at the beginning of each breath hold and displacements within a

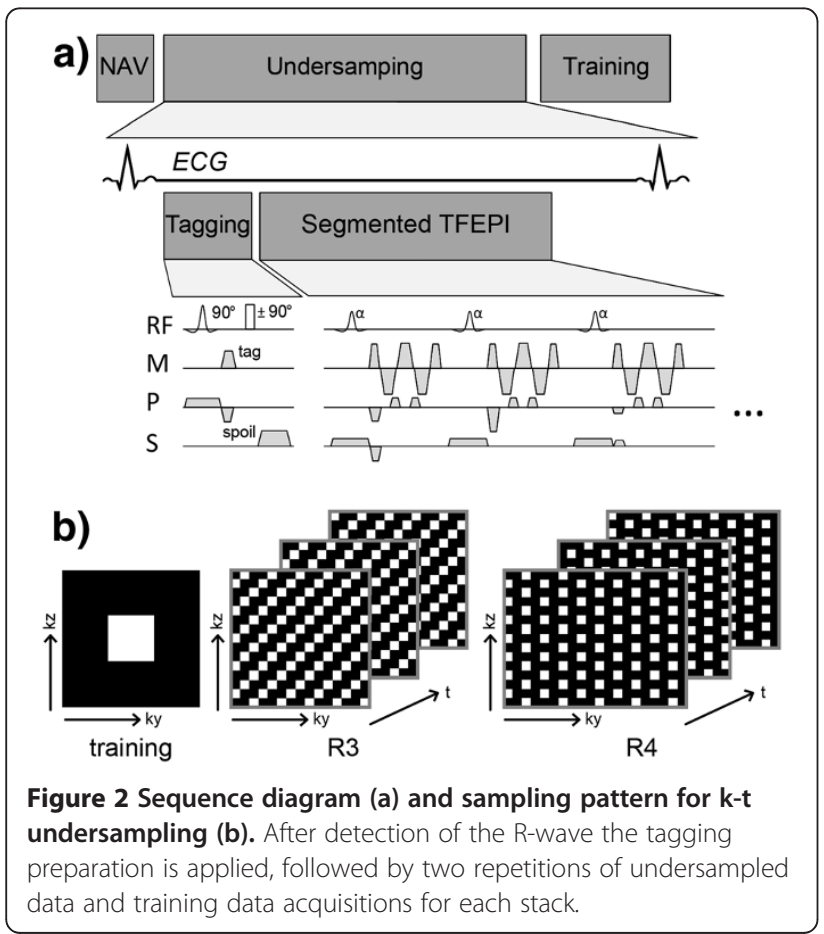

$5 \mathrm{~mm}$ gating window were accepted. The breath hold was repeated if the subject's breath hold level was not within the given window. Retrospective undersampling was applied with reduction factors of $\mathrm{R}=3$ and 4 . Prospectively undersampled data were acquired according to the schematic shown in Figure $2 \mathrm{~b}$ in an additional breath hold. To facilitate comparison, all imaging parameters except for undersampling factors were kept identical for fully sampled and undersampled data acquisitions. For undersampling factors $\mathrm{R}=3$ and 4 the corresponding sets of parameters are given in Table 2. Prior to image reconstruction, the 5-channel coil array data were compressed into a single virtual coil data set using the array compression method proposed by Buehrer et al. [45].

\section{Data analysis}

Epicardial and endocardial contours were manually drawn for each slice of interest and a midmural contour was calculated. This contour was tracked over time using the peak combination harmonic phase (HARP) $[46,47]$ algorithm implemented in an in-house software. Rotational motion and circumferential shortening was

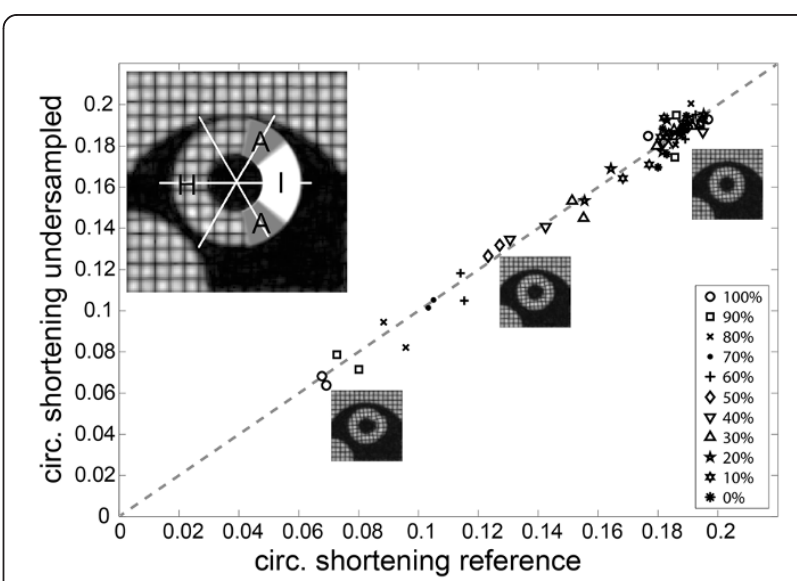

Figure 3 Comparison of peak circumferential shortening from undersampled $(R=3)$ and fully sampled simulated data. Myocardium was divided into three groups: infarction (I), which covers a sector of $80^{\circ}$ (22\% of myocardium), healthy tissue $(\mathrm{H})$ and infarct adjacent (A) tissue "attaching" infarcted to healthy tissue over a sector of $40^{\circ}$ (11\% of myocardium). For infarcted tissue, different degrees of immobilized tissue ranging from $0 \%$ to $100 \%$ were simulated. The dashed line indicates the reference diagonal with slope 1. 
Table 2 Imaging parameters for the acquisition of fully sampled reference and undersampled data $(R=3$ and 4$)$

\begin{tabular}{|c|c|c|}
\hline Undersampling factor & Fully sampled / R3 & Fully sampled / R4 \\
\hline Matrix size $k_{x} \times k_{y}$ & $28 \times 15$ & $28 \times 20$ \\
\hline Phase-encodes $k_{z}$ & 15 & 15 \\
\hline Receiver bandwidth & $314 \mathrm{~Hz} /$ pixel & $314 \mathrm{~Hz} /$ pixel \\
\hline Spatial resolution & $3.8 \times 7.2 \times 7.2 \mathrm{~mm}^{3}$ & $3.8 \times 5.4 \times 7.2 \mathrm{~mm}^{3}$ \\
\hline Heart phases & 21 & 24 \\
\hline Temporal resolution & $30.3 \mathrm{~ms}$ & $30.3 \mathrm{~ms}$ \\
\hline EPI factor & 5 & 5 \\
\hline TFE factor & 5 & 5 \\
\hline TE/TR & $2.8 \mathrm{~ms} / 6.1 \mathrm{~ms}$ & $2.8 \mathrm{~ms} / 6.1 \mathrm{~ms}$ \\
\hline R-R intervals undersampled $_{\text {. }}$ & 18 & 18 \\
\hline$\overline{R_{\text {net }}}$ & 2.25 & 3 \\
\hline Training profiles $\left(k_{y}, k_{z}\right)$ & $25(5 / 5)$ & $25(5 / 5)$ \\
\hline $\mathrm{R}-\mathrm{R}$ intervals $s_{\text {training }}$ & 6 & 6 \\
\hline R-R intervals full $_{\text {f }}$ & $3 \times 18$ & $3 \times 18$ \\
\hline Tag line distance & $7 \mathrm{~mm}$ & $7 \mathrm{~mm}$ \\
\hline
\end{tabular}

calculated from tracked contours as previously described by Ryf et al. [48]. Curves of circumferential shortening and rotation over the cardiac cycle were fitted by $8^{\text {th }}$ order polynomials, and maxima were found by estimating roots of the derivative. From the mean rotation over the contour, torsion was derived by taking the difference in peak rotation at the most apical and the most basal level [49]. Analysis of peak circumferential shortening and rotation was performed in six sectors per slice in 9 slices for computer simulation and 8-9 slices in in-vivo experiments. Comparisons were performed sector-wise and are reported for the entire LV.

For simulation the tracking results from fully sampled data were used as ground truth reference. For in-vivo measurements the mean of paired data points was used as reference. In order to facilitate comparison the initial contour was kept the same for the fully sampled reference and the retrospectively undersampled data in simulation and in-vivo. In the comparison of fully sampled data with prospectively undersampled acquisitions, contours were redrawn to account for changes in breath hold position and/or patient motion in-between scans.

Analysis of simulated data was done by calculating the relative difference defined as: error $_{r e l}=$ $\left|\max \left(\operatorname{motion}_{R=1}\right)-\max \left(\operatorname{motion}_{R>1}\right)\right| / \mid \max \left(\operatorname{motion}_{R=1}\right)-$ $\min \left(\operatorname{motion}_{R=1}\right) \mid$. The fully sampled data was used as reference. Relative differences \pm one standard deviation are reported in \% for circumferential shortening and rotation. In order to estimate the correlation between undersampled and reference data linear regression was performed correlating circumferential shortening and rotation of reference data and undersampled data. Regression slope, offset and the corresponding 95\% confidence interval were estimated as well as the correlation coefficient $R^{2}$ and the standard error of the estimate (SEE). The SEE is given in \% of the range of motion and is defined as $S E E=\sqrt{\frac{1}{N-2} \sum_{i=1}^{N}\left(x_{i}-x^{\prime}\right)^{2}}$ with $N$ being the number of points, $x_{i}$ denoting measurement data and $x_{i}^{\prime}$ corresponding values derived from linear regression. For in-vivo imaging, peak circumferential shortening and rotation were compared using Bland Altman analysis. Mean differences and the 95\% levels of agreement corresponding to 2 standard deviations (2SD) are reported. Linear regression was performed on peak circumferential shortening and rotation. Regression slopes, offsets, correlation coefficients and the SEE are reported.

Comparing reference data and retrospectively undersampled data, the HARP tracking performance of contour coordinates was studied based on contour vertex definitions in polar coordinates. Similar to the analysis of peak circumferential shortening and rotation, Bland Altman and linear regression analysis were performed.

Patient data are reported using bull's-eye plots of peak circumferential shortening, and late gadolinium enhancement (LGE). The centre of the bull's-eye plots represents the apex and the outer ring the base of the LV. For LGE images the average myocardial signal intensity per slice and sector normalized to the signal intensity measured in infarcted tissue is shown. Sectors were grouped into two groups: 1) non-viable sectors having more than $50 \%$ of the area presenting LGE and 2) viable sectors having less or equal to $50 \%$ of the area presenting LGE. Peak circumferential shortening was estimated for all sectors in each group and the median, $50^{\text {th }}$ percentiles and $90^{\text {th }}$ percentiles were estimated and presented in box-plots. Statistical significance of the differences in peak circumferential shortening between both groups were estimated by a two-tailed Wilcoxon signed-rank test. The results were Bonferroni corrected for repeated testing. A p-value less than 0.05 was considered statistically significant.

\section{Results}

\section{Computer simulation}

Normalized differences in peak circumferential shortening and rotation between undersampling factors of $R=3$, 4,5 and 8 and fully sampled data used as reference are shown in Figure 4a-b. Errors in circumferential shortening and rotation were below $5 \%$ for undersampling factors of $\mathrm{R}=3-5$ and $\mathrm{R}=3,4$, respectively. At $\mathrm{R}=8$ errors in circumferential shortening and rotation were 2.5 and 3.1 times greater compared to data obtained with $R=4$. The differences in torsion between undersampled and fully sampled data were $-0.13^{\circ}, 0.42^{\circ}, 0.95^{\circ}$ and $1.85^{\circ}$ for $\mathrm{R}=3,4,5$ and 8 , respectively. 
Figure $4 \mathrm{c}-\mathrm{j}$ shows regression slope, offset, correlation coefficient and SEE for $\mathrm{R}=3,4,5$ and 8 . The 95\% confidence intervals are presented as error bars for fitted slopes and offsets.

Figure 3 shows the result of motion tracking in the presence of simulated infarction. Peak circumferential shortening in an equatorial slice is plotted for the reference vs. undersampled data. Linear regression was performed, resulting in a slope of $1.01 \quad( \pm 0.04 \quad 95 \%$ confidence interval) and an offset of $-2.01 \%$ of the range of contraction $( \pm 4.79 \% 95 \%$ confidence interval).

\section{In-vivo measurements}

Figure 5 shows short axis slices reconstructed from fully sampled and 3- and 4-fold retrospectively and prospectively undersampled data. Frames at $27 \mathrm{~ms}$ after detection

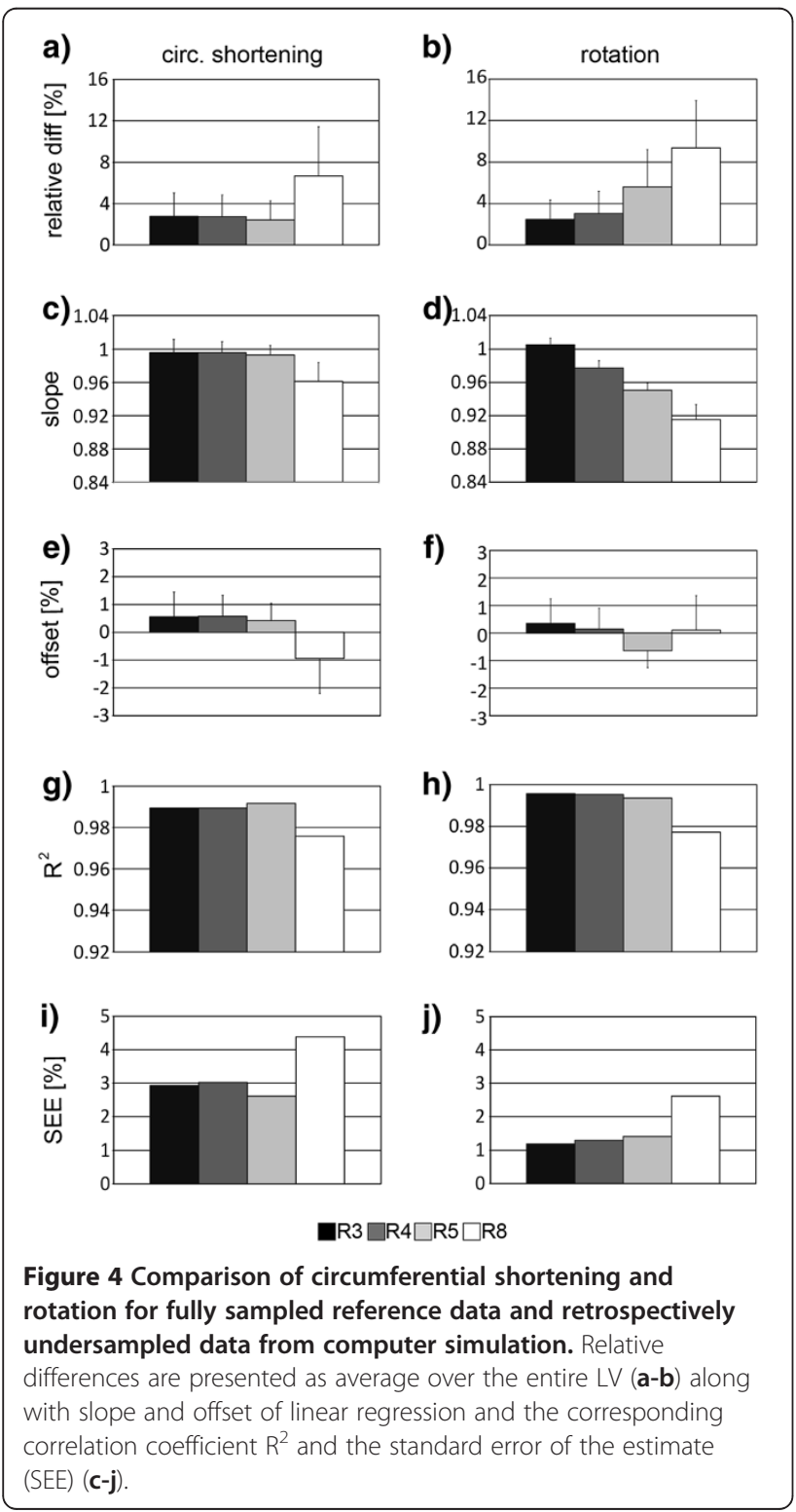

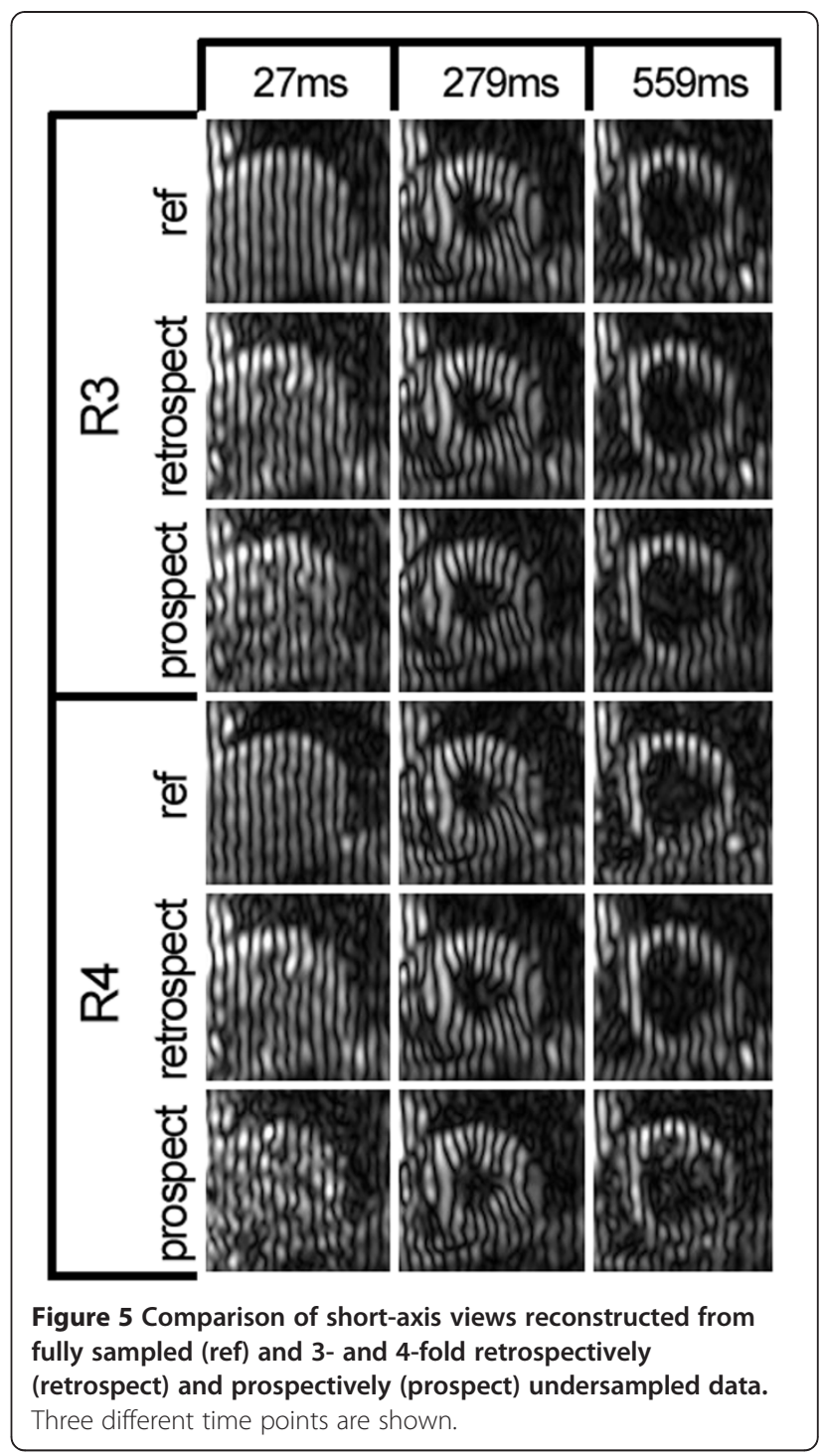

of the R-wave, at end systole (279 ms) and at mid diastole (559 $\mathrm{ms}$ ) are shown.

Linear regression analysis performed on fully sampled and retrospectively undersampled data is shown in Figure 6. For each regression the equations of the linear fit, the correlation coefficients $\mathrm{R}^{2}$ and the SEE are given. Figure 6a-d show the analysis of polar coordinates of tracked points and Figure 6e-h demonstrate sector-wise comparison of time curves of circumferential shortening and rotation for $\mathrm{R}=3$ and 4 .

In Figure 7 shows the Bland-Altman comparison of fully sampled and retrospectively undersampled in-vivo data for $R=3$ and 4. Dashed lines represent the mean difference (light grey) and the 95\% limit of agreement (2SD) (black). In Figure 7a-d provides the comparison of radial coordinates $(r=$ radius and $\phi=$ angle) of tracked contour points. Mean differences $\pm 2 \mathrm{SD}$ of $\mathrm{r}$ were $0.0 \pm 3.3 \%$ and $-0.2 \pm 4.1 \%$ for $\mathrm{R}=3$ and 4 and $-0.2 \pm 1.9^{\circ}$ 

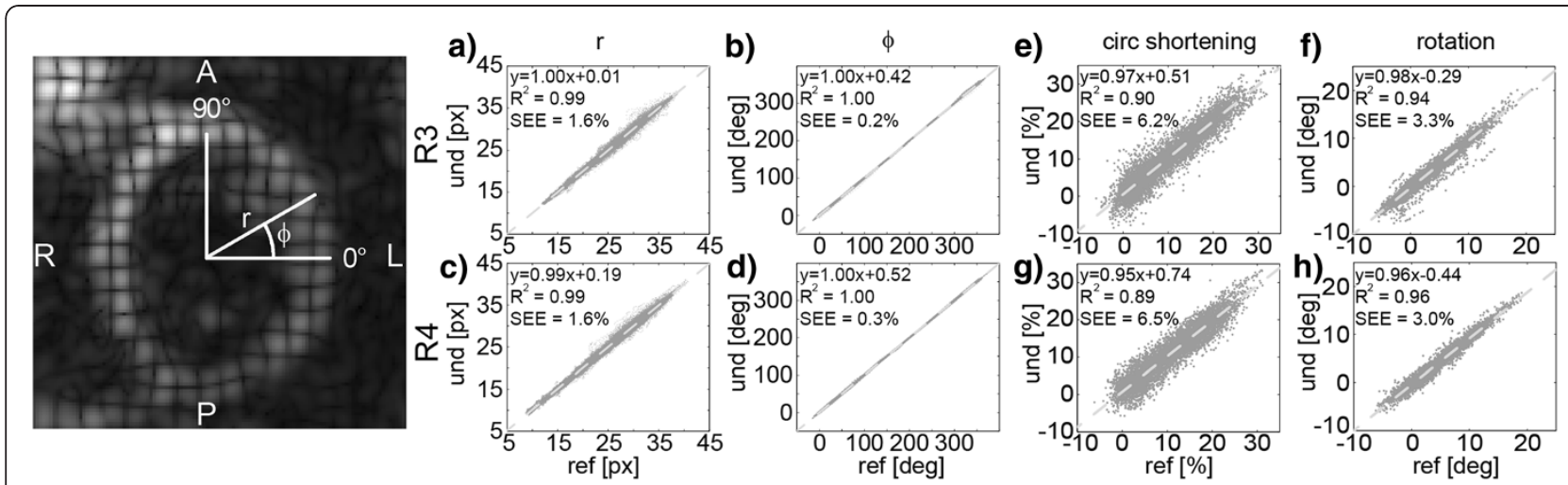

Figure 6 Linear regression analysis for point-wise and sector-wise comparison of fully sampled (ref) and retrospectively undersampled (und) data in healthy volunteers ( $R=\mathbf{3}$ and $\mathbf{4})$. Point-wise comparison was performed for polar coordinates $r(\mathbf{a}, \mathbf{c})$ and $\varphi$ (b, $\mathbf{d})$. For circumferential shortening $(\mathbf{e}, \mathbf{g})$ and rotation $(\mathbf{f}, \mathbf{h})$ entire motion curves were compared per sector across the entire LV.

and $-0.3 \pm 2.2^{\circ}$ for $\phi$. In Figure $7 \mathrm{e}-\mathrm{h}$ Bland-Altman plots for peak circumferential shortening and peak rotation are given. The mean differences $\pm 2 \mathrm{SD}$ for circumferential shortening were $-0.2 \pm 4.1 \%$ and $-0.1 \pm 4.2 \%$ for $\mathrm{R}=3$ and 4. For rotation, mean differences $\pm 2 \mathrm{SD}$ were $0.5 \pm 1.8^{\circ}$ and $0.7 \pm 1.7^{\circ}$ for $\mathrm{R}=3$ and 4 . The mean differences of torsion were $0.45 \pm 2.22^{\circ} \quad(p=$ N.S. $)$ and $0.05 \pm 2.24^{\circ}$ ( $\mathrm{p}=$ N.S.) for $\mathrm{R}=3$ and 4 , respectively.

Figure 8 shows the resulting Bland-Altman analysis of peak motion $(\mathrm{a}-\mathrm{d})$ and correlation between full time curves of motion (e-h) comparing fully sampled data with data acquired with prospective undersampling $(\mathrm{R}=3$ and 4$)$. Mean differences $\pm 2 \mathrm{SD}$ of peak circumferential shortening were $-0.1 \pm 5.2 \%$ and $-0.7 \pm 6.2 \%$ for $\mathrm{R}=3$ and 4 . Mean differences of peak rotation were found to be $0.44 \pm 1.80^{\circ}$ and $0.73 \pm 1.67^{\circ}$ for $\mathrm{R}=3$ and 4 while differences in torsion were $0.48 \pm 4.20^{\circ}$ ( $\mathrm{p}=\mathrm{N} . \mathrm{S}$.) and $0.03 \pm 4.48^{\circ}$ ( $\mathrm{p}=$ N.S.) for $\mathrm{R}=3$ and 4 , respectively.

Bull's-eye plots of peak circumferential shortening and profiles of circumferential shortening and rotation derived from undersampled $(\mathrm{R}=4)$ and fully sampled data are compared in Figure 9 for one healthy subject. Spatially depend differences are not observed.

Figure 10 compares circumferential shortening obtained from undersampled and fully sampled reference data for the five patients in relation the LGE findings. Differences in peak circumferential shortening in sectors with more than $50 \%$ of their area presenting LGE and sectors with less than 50\% LGE were statistically significant in all patients. Mean difference $\pm 2 S D$ in torsion between undersampled and fully sampled reference data in patients was $0.72 \pm 2.14^{\circ}$.
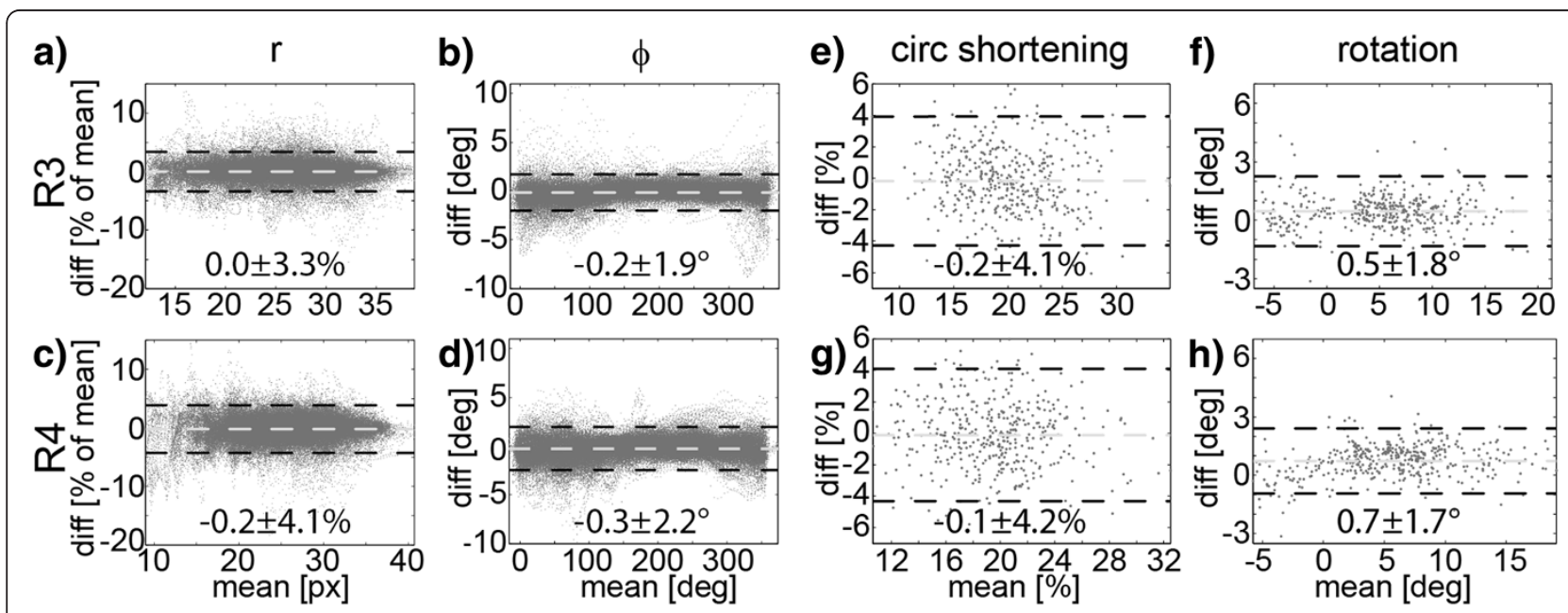

Figure 7 Bland Altman plots of point-wise and sector-wise comparison of fully sampled and retrospectively undersampled in-vivo data ( $\mathbf{R = 3}$ and $\mathbf{4})$ in healthy volunteers. Mean differences (grey) and 95\% levels of agreement (2SD) (black) are indicated by the dashed lines. Analysis was performed on the entire LV. Point-wise comparison was performed for the polar coordinates $r(\mathbf{a}, \mathbf{c})$ and $\varphi(\mathbf{b}, \mathbf{d})$ for each tracked point. Circumferential shortening $(\mathbf{e}, \mathbf{g})$ and rotation $(\mathbf{f}, \mathbf{h})$ were compared per sector. 


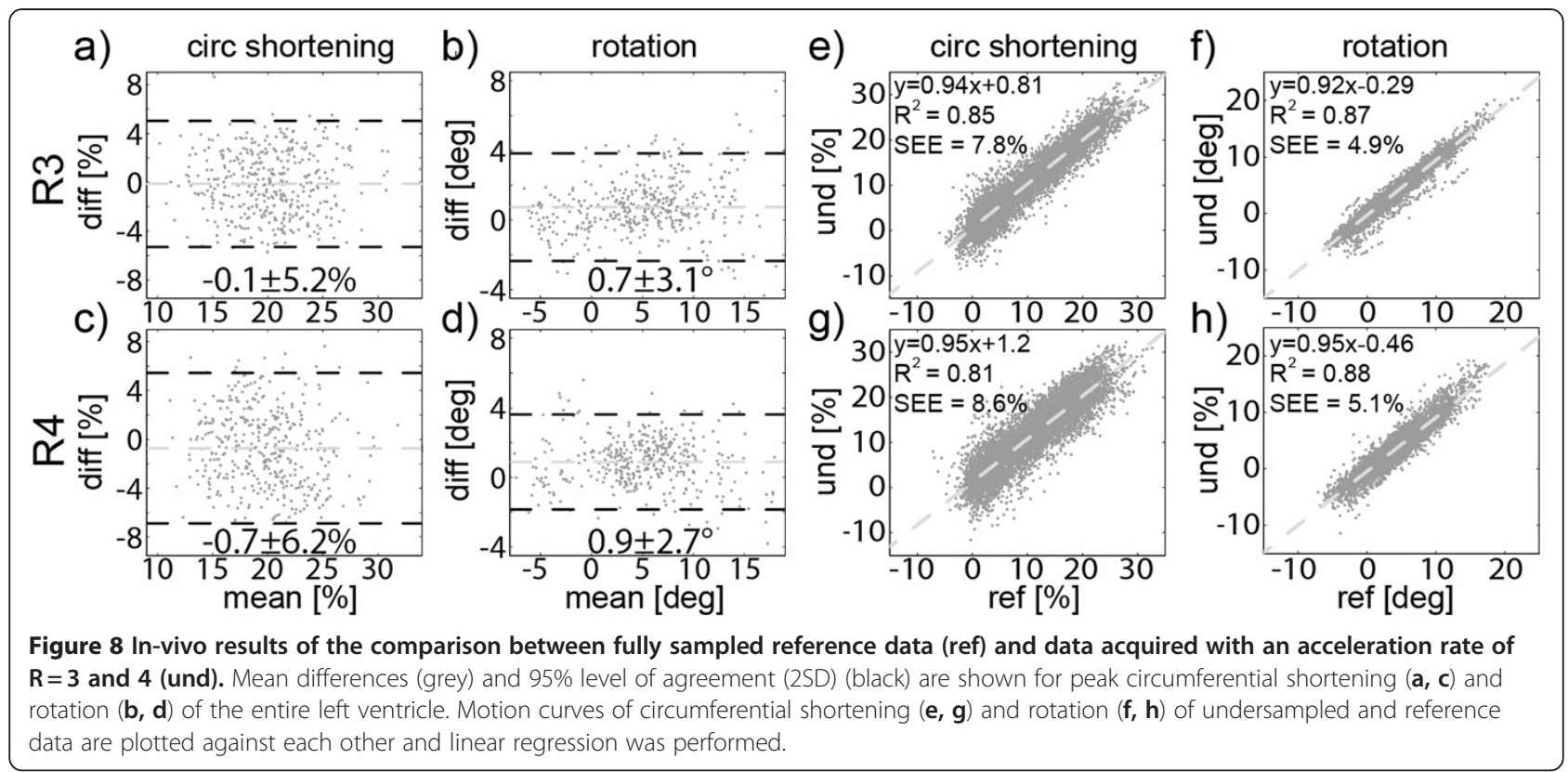

The SNR in the fully sampled in-vivo data was $36 \pm 12$ on average.

\section{Discussion}

In this work undersampled 3D CSPAMM tagging in combination with $\mathrm{k}$-t PCA has been implemented and validated.
The analysis of simulation results has revealed a maximum applicable acceleration factor of four. Generally, good correlation was found between fully sampled and retrospectively undersampled data over the entire left ventricle. Analysis of relative differences in circumferential shortening showed a slight decrease in error going from the apex towards the base of the heart. As motion

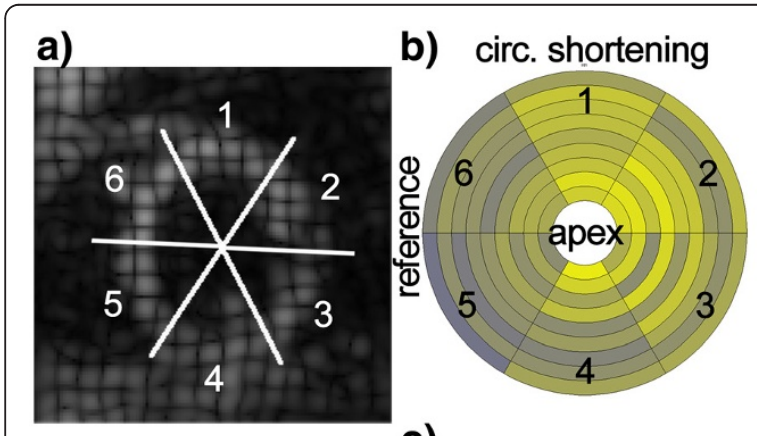

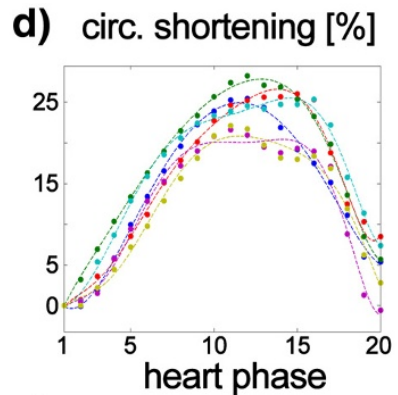

e)

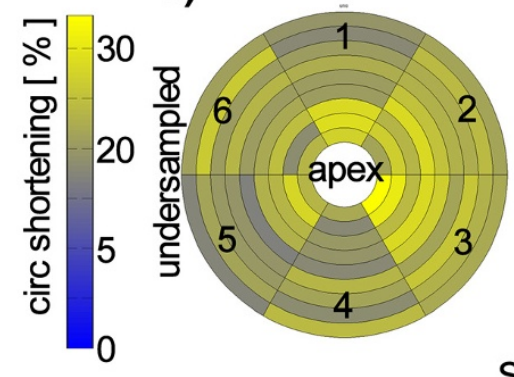

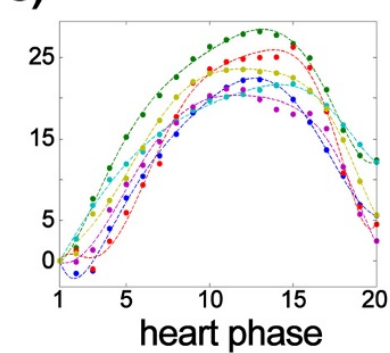

Sector 1 Sector 2 Sector 3

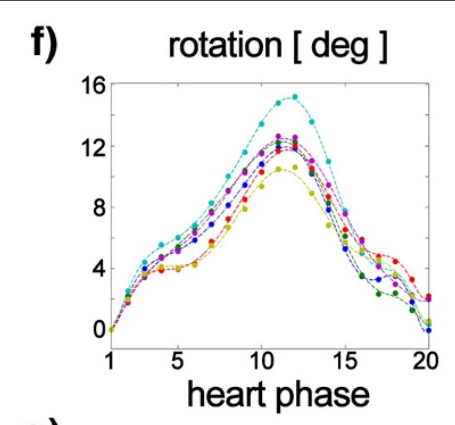

g)

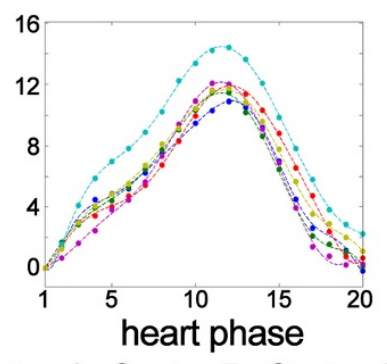

Figure 9 Comparison of circumferential shortening for fully sampled data and 4-fold undersampled data. The first column shows maps of peak circumferential shortening for fully sampled $\mathbf{b}$ ) and undersampled data $\mathbf{c}$ ). Resulting motion profiles taken from an equatorial slice are shown for circumferential shortening $(\mathbf{d}, \mathbf{e})$ and rotation $(\mathbf{f}, \mathbf{g})$. The dots represent the actual data points while the line represents the polynomial fit. 

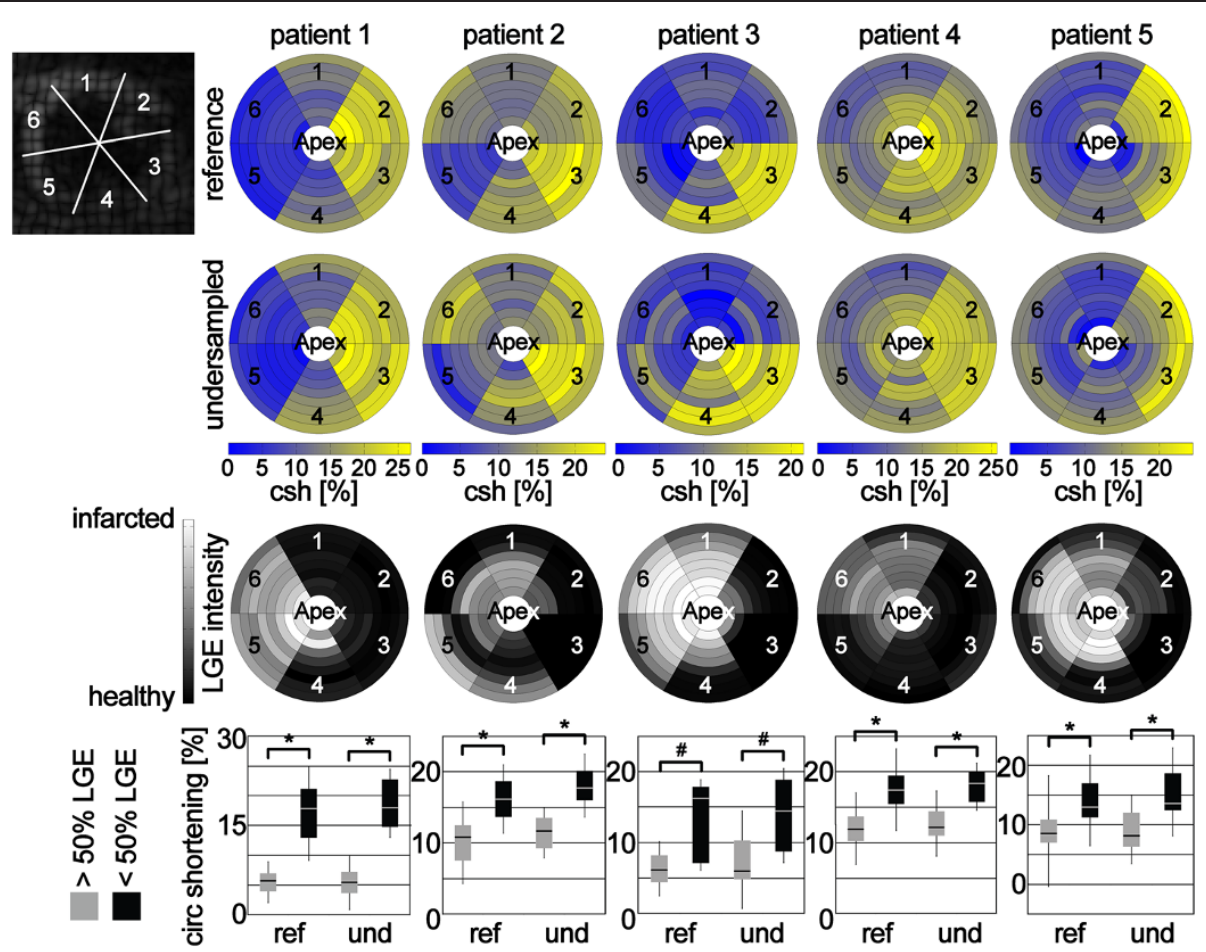

Figure 10 Maps of peak circumferential shortening (csh) for five patients with myocardial infarction. The top row represents results from fully sampled data while the second row shows data from prospectively undersampled acquisitions. The third row shows the result of late gadolinium enhancement imaging (LGE). The myocardial signal intensity post contrast was averaged over each sector and normalized to the signal intensity from completely infarcted tissue. The bottom row shows box-plots of peak circumferential shortening for sectors with more than $50 \%$ late gadolinium enhanced signal (grey) and sectors with less than 50\% late gadolinium enhanced signal (black). The box represents $50 \%$ of the data points and the error bars $90 \%$. Statistical significance is indicated by \# for $p<0.002$ and * for $p<0.0002$.

was normalized for each slice, this observation is associated with the low magnitude of contraction of apical myocardium.

Different transmural extensions of infarcted tissue were investigated by computer simulation. Motion analysis showed a good correlation between undersampled and fully sampled reference data. Transmural reduction of contraction as low as $20 \%$ could be distinguished from healthy fully contracting tissue. Although fully infarcted tissue was simulated as well, sector-wise analysis always showed contraction greater than $6 \%$. This is due to the choice of the position and size of the sectors used for analysis. Sectors with non-contracting tissue contained slightly contracting neighbouring tissue, which leads to a non-zero average circumferential shortening within a sector.

In vivo comparison between fully sampled data and retrospectively undersampled data showed good agreement as the mean difference was less than $1 \%$ (\% of range of motion). Differences between reference data and prospectively undersampled acquisition were found to be larger in comparison to differences seen relative to retrospectively undersampled data. This finding is related to multiple issues. On one hand, reference data and undersampled data were acquired in two consecutive scans. Despite the use of a gating respiratory navigator, different breath hold levels are possible and slices used for analysis might hence differ in position. On the other hand the fully sampled reference data itself was acquired in three consecutive breath holds, which, despite the use of a respiratory gating window of $5 \mathrm{~mm}$, can lead to stack misalignment within the reference data. The resulting circumferential shortening is dependent on the transmural position of the tracked contour. If stack misalignment occurs a contour that appears to be midmural in one stack can be positioned more epicardially or endocardially in the consecutive two stacks from which the motion orthogonal to the first stack is derived from. Hence a contour point is tracked at three different transmural positions. Therefore the motion profiles obtained from fully sampled data, used as gold standard reference in this work, might have been compromised. Image registration was not performed in this study. The implementation of tailored registration algorithms that can handle orthogonal tagging modulations requires further investigation. 
Maps of peak circumferential shortening as well as the corresponding motion profiles were found to agree well for reference and undersampled data. The magnitude of circumferential shortening is comparable to previously reported values $[24,25,50,51]$. Besides potential motion smoothing, expressed by the positive mean difference for rotation, a slice by slice comparison of prospectively undersampled and fully sampled data might have been biased by an offset in breath hold level for both acquisitions.

In this study, standard 2D single slice tagging data were not available for reference. In order to capture through-plane motion and hence make data comparable to 3D tagging, non-standard extensions of 2D tagging such as slice following [15] or zHARP [18] are required. Accordingly, assessment of error in the present work was relative to fully sampled 3D CSPAMM data only, which is a clear limitation. Nevertheless, there have been previous records of validating 3D CSPAMM on healthy subjects and patients, which may serve as benchmark [24,25].

Spatial resolution in each of the three orthogonal stacks in 3D CSPAMM may be considered coarse $\left(3.8 \times 5.4-7.2 \times 7.2 \mathrm{~mm}^{3}\right)$. The resolution given in readout direction is, however, directly linked to the tagline spacing when using HARP analysis. Higher temporal resolution $(<30 \mathrm{~ms})$ is desirable as this allows separating data in $\mathrm{x}$-pc space further and hence improves reconstruction accuracy in $\mathrm{k}-\mathrm{t}$ PCA. However, this results in more heart phases and hence more RF excitations per cardiac cycle reducing the contrast-to-noise ratio of the tagged data.

Five patients with myocardial infarction were examined using undersampled tagging and LGE imaging. Maps of circumferential shortening derived from reference data and prospectively undersampled data agreed well and infarcted regions could be localized. Direct correlation of peak circumferential shortening with the area of delayed enhancement was found to be only moderate. A potential limitation of infarct detection is related to averaging of motion within sectors. A six-sector per slice model $[24,25]$ results in $16.7 \%$ of total myocardial mass captured per sector and per slice. For example, nonviable tissue in border zones of infarction is passively moved and compressed and hence the extent of motion abnormality may be overestimated. This issue may be addressed by increasing the number of sectors per slice and by using multiple circumferential contours covering the entire transmural extent in future work.

Several strategies may be envisioned to shorten the relatively long breath hold durations $(\sim 20 \mathrm{sec})$ of the present protocols. First, sampling of training data can be fully integrated into the acquisition of undersampled data. Such a variable density EPI approach provides shorter overall scan duration at the expense of reduced temporal resolution. Second, the separation of training data and undersampled data allows splitting the data acquisition into two breath holds. Compared to the method proposed by Rutz et al. [24] the three stacks are not acquired within three consecutive breath holds, but could be acquired within one breath hold for high resolution undersampled and a second breath hold for low resolution training data. Since the training data has very low spatial resolution in the phase-encode directions $\left(2.3 \times 2.3 \mathrm{~cm}^{2}\right)$, differences in breath hold levels between acquisitions no longer pose a concern.

\section{Conclusion}

Undersampled cine 3D tagging in conjunction with $\mathrm{k}-\mathrm{t}$ PCA reconstruction enables significant reduction in scan time of whole-heart tagging and facilitates efficient and accurate quantification of shortening, rotation and torsion of the left ventricle. Using 3-fold undersampling the entire cine 3D tagging acquisition could be accommodated in a single breath hold and feasibility in volunteers and patients was demonstrated. Future work is dedicated to shorten breath hold durations further and to apply the method in larger patient cohorts to prove clinical value.

\section{Abbreviations}

CMR: Cardiovascular magnetic resonance; MI: Myocardial infarction; CAD: Coronary artery disease; LBBB: Left bundle branch block; CRT: Cardiac resynchronization therapy; LV: Left ventricle; PCI: Percutaneous coronary intervention; LGE: Late-gadolinium enhancement; DENSE: Displacement encoding; SPAMM: Spatial modulation of magnetization; CSPAMM: Complementary spatial modulation of magnetization; HARP: Harmonic phase; PCA: Principal component analysis; SNR: Signal to noise ratio; SEE: Standard error of the estimate; ref: Reference; und: Undersampling; csh: Circumferential shortening.

\section{Competing interests}

The authors declare that they have no competing interest.

\section{Authors' contributions}

CS: study design, data acquisition, reconstruction, image analysis, manuscript drafting, statistics, and literature research. RM: study design, image analysis, statistics and revision of manuscript. PB: study design, literature research, and revision of manuscript. SK: Study design, reconstruction, literature research and revision of manuscript. All authors read and approved the final manuscript.

\section{Acknowledgments}

The authors acknowledge funding by the Swiss National Science Foundation, grant \#CR3213_132671/1.

\section{Author details}

${ }^{1}$ Institute for Biomedical Engineering, University and ETH Zurich, Gloriastrasse 35, 8092 Zurich, Switzerland. 'Department of Cardiology, University Hospital, Zurich, Switzerland. ${ }^{3}$ maging Sciences and Biomedical Engineering, King's

College London, London, United Kingdom.

Received: 26 February 2012 Accepted: 2 August 2012

Published: 30 August 2012

\section{References}

1. Bilchick KC, Lardo AC: Cardiac resynchronization therapy: application of imaging to optimize patient selection and assess response. Curr Heart Fail Rep. 2008, 5:119-27. 
2. Jehle A, Epstein F, Zhong X, Janiczek R, Tsai WK, Christopher J, Fowler D, Ferguson J, Kramer C, Bilchick K: Cine DENSE MRI for circumferential and radial dyssynchrony in patients referred for cardiac resynchronization therapy. J Cardiovasc Magn Reson. 2009, 11:090.

3. Nagel E, Stuber M, Lakatos M, Scheidegger MB, Boesiger P, Hess OM: Cardiac rotation and relaxation after anterolateral myocardial infarction. Coron Artery Dis. 2000, 11:261-7.

4. Stuber M, Scheidegger MB, Fischer SE, Nagel E, Steinemann F, Hess OM, Boesiger $P$ : Alterations in the local myocardial motion pattern in patients suffering from pressure overload due to aortic stenosis. Circulation. 1999, 100:361-8.

5. Sandstede JJ, Johnson T, Harre K, Beer M, Hofmann S, Pabst T, Kenn W, Voelker W, Neubauer S, Hahn D: Cardiac systolic rotation and contraction before and after valve replacement for aortic stenosis: a myocardial tagging study using MR imaging. AJR Am J Roentgenol. 2002, 178:953-8.

6. McVeigh ER: MRI of myocardial function: motion tracking techniques. Magn Reson Imaging. 1996, 14:137-50.

7. Gotte MJ, Germans T, Russel IK, Zwanenburg JJ, Marcus JT, van Rossum AC, van Veldhuisen DJ: Myocardial strain and torsion quantified by cardiovascular magnetic resonance tissue tagging: studies in normal and impaired left ventricular function. J Am Coll Cardiol. 2006, 48:2002-11.

8. Aletras AH, Ding S, Balaban RS, Wen H: DENSE: displacement encoding with stimulated echoes in cardiac functional MRI. J Magn Reson. 1999, 137:247-52.

9. Spottiswoode BS, Zhong X, Lorenz CH, Mayosi BM, Meintjes EM, Epstein FH: 3D myocardial tissue tracking with slice followed cine DENSE MRI. J Magn Reson Imaging. 2008, 27:1019-27.

10. Arai AE, Gaither CC 3rd, Epstein FH, Balaban RS, Wolff SD: Myocardial velocity gradient imaging by phase contrast MRI with application to regional function in myocardial ischemia. Magn Reson Med. 1999, 42:98-109.

11. Robson MD, Constable RT: Three-dimensional strain-rate imaging. Magn Reson Med. 1996, 36:537-46.

12. Jung B, Zaitsev M, Hennig J, Markl M: Navigator gated high temporal resolution tissue phase mapping of myocardial motion. Magn Reson Med. 2006, 55:937-42.

13. Axel L, Dougherty L: MR Imaging of Motion with Spatial Modulation of Magnetization. Radiology. 1989, 171:841-5.

14. Fischer SE, McKinnon GC, Maier SE, Boesiger P: Improved myocardial tagging contrast. Magn Reson Med. 1993, 30:191-200.

15. Stuber M, Spiegel MA, Fischer SE, Scheidegger MB, Danias PG, Pedersen EM, Boesiger P: Single breath-hold slice-following CSPAMM myocardial tagging. MAGMA. 1999, 9:85-91.

16. Fischer SE, McKinnon GC, Scheidegger MB, Prins W, Meier D, Boesiger P: True myocardial motion tracking. Magn Reson Med. 1994, 31:401-13.

17. Moore CC, O'Dell WG, MCVeigh ER, Zerhouni EA: Calculation of threedimensional left ventricular strains from biplanar tagged MR images. J Magn Reson Imaging. 1992, 2:165-75.

18. Abd-Elmoniem KZ, Stuber M, Osman NF, Prince JL: ZHARP: threedimensional motion tracking from a single image plane. Inf Process Med Imaging. 2005, 19:639-51.

19. Abd-Elmoniem KZ, Stuber M, Prince JL: Direct three-dimensional myocardial strain tensor quantification and tracking using zHARP. Med Image Anal. 2008, 12:778-86.

20. Ryf S, Spiegel MA, Gerber M, Boesiger P: Myocardial tagging with 3DCSPAMM. J Magn Reson Imaging 2002, 16:320-5.

21. Ryf S, Baltes C, Boesiger P: High speed 3D CSPAMM. Kyoto, Japan: 12th Annual Meeting of ISMRM; 2004: p. 657.

22. Zhong X, Spottiswoode BS, Meyer CH, Kramer CM, Epstein FH: Imaging three-dimensional myocardial mechanics using navigator-gated volumetric spiral cine DENSE MRI. Magn Reson Med. 2010, 64:1089-97.

23. Zhong X, Spottiswoode B, Meyer C, Kramer C, Epstein F: Navigator-gated 3D cine DENSE: development and initial evaluation. J Cardiovasc Magn Reson. 2009, 11:094.

24. Rutz AK, Ryf S, Plein S, Boesiger P, Kozerke S: Accelerated whole-heart 3D CSPAMM for myocardial motion quantification. Magn Reson Med. 2008 59:755-63.

25. Rutz AK, Manka R, Kozerke S, Roas S, Boesiger P, Schwitter J: Left ventricular dyssynchrony in patients with left bundle branch block and patients after myocardial infarction: integration of mechanics and viability by cardiac magnetic resonance. Eur Heart J. 2009, 30:2117-27.
26. Wang Y, Riederer SJ, Ehman RL: Respiratory motion of the heart: kinematics and the implications for the spatial resolution in coronary imaging. Magn Reson Med. 1995, 33:713-9.

27. Nehrke K, Bornert P, Manke D, Bock JC: Free-breathing cardiac MR imaging: study of implications of respiratory motion-initial results. Radiology. 2001, 220:810-5.

28. Moghari MH, Hu P, Kissinger KV, Goddu B, Goepfert L, Ngo L, Manning WJ, Nezafat R: Subject-specific estimation of respiratory navigator tracking factor for free-breathing cardiovascular MR. Magn Reson Med. 2012, 67:1665-72.

29. Lustig M, Donoho D, Pauly JM: Sparse MRI: The application of compressed sensing for rapid MR imaging. Magn Reson Med. 2007, 58:1182-95.

30. Tsao J, Boesiger P, Pruessmann KP: k-t BLAST and k-t SENSE: dynamic MRI with high frame rate exploiting spatiotemporal correlations. Magn Reson Med. 2003, 50:1031-42.

31. Pedersen $H$, Kozerke $S$, Ringgaard $S$, Nehrke $K$, Kim WY: k-t PCA: temporally constrained k-t BLAST reconstruction using principal component analysis. Magn Reson Med. 2009, 62:706-16.

32. Bo Z, Haldar JP, Brinegar C, Zhi-Pei L: Low rank matrix recovery for realtime cardiac MRI. In Biomedical Imaging: From Nano to Macro, 2010 IEEE International Symposium. 2010: p. 996-9.

33. Gamper $U$, Boesiger $P$, Kozerke $S$ : Compressed sensing in dynamic MRI. Magn Reson Med. 2008, 59:365-73.

34. Plein S, Ryf S, Schwitter J, Radjenovic A, Boesiger P, Kozerke S: Dynamic contrast-enhanced myocardial perfusion MRI accelerated with k-t sense. Magn Reson Med. 2007, 58:777-85.

35. Otazo R, Kim D, Axel L, Sodickson DK: Combination of compressed sensing and parallel imaging for highly accelerated first-pass cardiac perfusion MRI. Magn Reson Med. 2010, 64:767-76.

36. Jung B, Honal M, Hennig J, Markl M: k-t-Space accelerated myocardial perfusion. J Magn Reson Imaging. 2008, 28:1080-5.

37. Adluru G, McGann C, Speier P, Kholmovski EG, Shaaban A, Dibella EV: Acquisition and reconstruction of undersampled radial data for myocardial perfusion magnetic resonance imaging. J Magn Reson Imaging. 2009, 29:466-73.

38. Manka R, Vitanis V, Boesiger P, Flammer AJ, Plein S, Kozerke S: Clinical feasibility of accelerated, high spatial resolution myocardial perfusion imaging. JACC Cardiovasc Imaging. 2010, 3:710-7.

39. Manka R, Jahnke C, Kozerke S, Vitanis V, Crelier G, Gebker R, Schnackenburg $B$, Boesiger $P$, Fleck E, Paetsch I: Dynamic 3-dimensional stress cardiac magnetic resonance perfusion imaging: detection of coronary artery disease and volumetry of myocardial hypoenhancement before and after coronary stenting. J Am Coll Cardiol. 2011, 57:437-44.

40. Hansen MS, Baltes C, Tsao J, Kozerke S, Pruessmann KP, Boesiger P, Pedersen EM: Accelerated dynamic Fourier velocity encoding by exploiting velocity-spatio-temporal correlations. MAGMA. 2004, 17:86-94.

41. Baltes C, Kozerke S, Hansen MS, Pruessmann KP, Tsao J, Boesiger P: Accelerating cine phase-contrast flow measurements using k-t BLAST and k-t SENSE. Magn Reson Med. 2005, 54:1430-8.

42. Stadlbauer A, van der Riet W, Crelier G, Salomonowitz E: Accelerated timeresolved three-dimensional MR velocity mapping of blood flow patterns in the aorta using SENSE and k-t BLAST. Eur J Radiol. 2010, 75:e15-21.

43. Peters DC, Epstein FH, McVeigh ER: Myocardial wall tagging with undersampled projection reconstruction. Magn Reson Med. 2001, 45:562-7.

44. Ryf S, Kozerke S, Tsao J, Boesiger P: 3D CSPAMM tagging accelerated with ktBLAST. Toronto, Canada: 11th Annual Meeting of ISMRM; 2003: p. 1565.

45. Buehrer M, Pruessmann KP, Boesiger P, Kozerke S: Array compression for MRI with large coil arrays. Magn Reson Med. 2007, 57:1131-9.

46. Osman NF, Kerwin WS, McVeigh ER, Prince JL: Cardiac motion tracking using CINE harmonic phase (HARP) magnetic resonance imaging. Magn Reson Med. 1999, 42:1048-60.

47. Ryf S, Tsao J, Schwitter J, Stuessi A, Boesiger P: Peak-combination HARP: a method to correct for phase errors in HARP. J Magn Reson Imaging. 2004, 20:874-80.

48. Ryf S, Rutz AK, Boesiger P, Schwitter J: Is post-systolic shortening a reliable indicator of myocardial viability? An MR tagging and late-enhancement study. J Cardiovasc Magn Reson. 2006, 8:445-51.

49. Nagel E, Stuber M, Burkhard B, Fischer SE, Scheidegger MB, Boesiger P, Hess $\mathrm{OM}$ : Cardiac rotation and relaxation in patients with aortic valve stenosis. Eur Heart J. 2000, 21:582-9. 
50. Kramer CM, Rogers WJ, Theobald TM, Power TP, Petruolo S, Reichek N: Remote noninfarcted region dysfunction soon after first anterior myocardial infarction. A magnetic resonance tagging study. Circulation. 1996, 94:660-6.

51. Rosen BD, Gerber BL, Edvardsen T, Castillo E, Amado LC, Nasir K, Kraitchman DL, Osman NF, Bluemke DA, Lima JA: Late systolic onset of regional LV relaxation demonstrated in three-dimensional space by MRI tissue tagging. Am J Physiol Heart Circ Physiol. 2004, 287:H1740-6.

doi:10.1186/1532-429X-14-60

Cite this article as: Stoeck et al.: Undersampled Cine 3D tagging for rapid assessment of cardiac motion. Journal of Cardiovascular Magnetic Resonance 2012 14:60.

\section{Submit your next manuscript to BioMed Central and take full advantage of:}

- Convenient online submission

- Thorough peer review

- No space constraints or color figure charges

- Immediate publication on acceptance

- Inclusion in PubMed, CAS, Scopus and Google Scholar

- Research which is freely available for redistribution 